Preprint typeset in JHEP style. - HYPER VERSION

CECS-PHY-06/20

\title{
Dilatation operator and the Super Yang-Mills duals of open strings on AdS Giant Gravitons
}

\author{
Diego H. Correa ${ }^{1, \dagger}$ and Guillermo A. Silva ${ }^{* 2 \ddagger}$ \\ 1 Centro de Estudios Cientifícos, Valdivia, Casilla 1469, Chile \\ ${ }^{2}$ Departamento de Física, Universidad Nacional de La Plata C.C. 67, 1900, \\ La Plata, Argentina \\ $\dagger$ dcorrea@cecs.cl \\ $\ddagger$ silva@fisica.unlp.edu.ar
}

\begin{abstract}
We study the one-loop anomalous dimensions of the Super Yang-Mills dual operators to open strings ending on AdS giant gravitons. AdS giant gravitons have no upper bound for their angular momentum and we represent them by the contraction of scalar fields, carrying the appropriate R-charge, with a totally symmetric tensor. We represent the open string motion along AdS directions by appending to the giant graviton operator a product of fields including covariant derivatives. We derive a bosonic lattice Hamiltonian that describes the mixing of these excited AdS giants operators under the action of the one-loop dilatation operator of $\mathcal{N}=4$ SYM. This Hamiltonian captures several intuitive differences with respect to the case of sphere giant gravitons. A semiclassical analysis of the Hamiltonian allows us to give a geometrical interpretation for the labeling used to describe the fields products appended to the AdS giant operators. It also allows us to show evidence for the existence of continuous bands in the Hamiltonian spectrum.
\end{abstract}

KEYWORDS: AdS/CFT, D-branes, open strings, spin chains.

*Associated with CONICET 


\section{Contents}

1. Introduction 1

2. Anomalous dimensions for the duals of open strings on AdS giants 3

2.1 The Semiclassical Limit 11

2.2 Continuous bands 14

3. Open strings on AdS giant gravitons 16

3.1 Semiclassical Open Strings 18

田. Discussion 21

丹. Conventions and some combinatorial properties 23

B. Open strings in the pp-wave limit 24

\section{Introduction}

The most accomplished realization of the AdS/CFT correspondence conjectures the equivalence between $\mathcal{N}=4$ Super Yang-Mills theory in four dimensions and type IIB string theory on the $A d S_{5} \times S^{5}$ background [1, 2, [3]. A great deal of evidence supporting this equivalence was found after the realization of a geometrical limit, for strings with large angular momentum, leading to a plane-wave background where the string theory can be exactly quantized [4, [5]. In this so called "BMN" limit, the closed strings energy spectrum was shown to match the scale dimension spectrum of certain dual operators (a.k.a. BMN operators) of the $\mathcal{N}=4$ gauge theory [6]. The key observation of Berenstein, Maldacena and Nastase [6] was to note that the perturbative contributions to the anomalous dimensions of BMN operators are suppressed by the square of the parameter associated with the angular momentum of the dual string. This fact allowed to engineer a limit in which perturbative computations on the gauge theory side could be extrapolated to strong coupling and therefore make a true comparison with string theory computations beyond the supergravity and BPS approximations.

Another remarkable result in the AdS/CFT large $N$ approximation ( $N$ being the rank of the gauge group) was the discovery of integrable structures governing the anomalous dimensions of $\mathcal{N}=4$ SYM operators. Minahan and Zarembo [7] showed 
that the mixing matrix of anomalous dimensions of single trace operators of scalar fields, at planar 1-loop approximation, is given by an integrable $\mathrm{SO}(6)$ spin chain Hamiltonian. More recently, the planar 1-loop anomalous dimensions for the full set of local operators were shown to be given by the spectrum of an integrable spin chain Hamiltonian for the full superconformal group [8].

Some of these ideas can be extended to the case where one includes D-branes and open strings. In these cases the spectrum of anomalous dimensions of the dual operators is given by open spin chain Hamiltonians. It is possible to work out the boundary conditions for the open spin chains and determine if they define integrable open spin chain models.

In [9], non-perturbative BPS states of finite energy on $A d S_{5} \times S^{5}$ were found by considering compact D3-branes expanded in a $S^{\prime 3} \subset S^{5}$. These spherical D-brane solutions, known as giant gravitons, are supersymmetric and it is also possible to construct them by wrapping a $S^{3} \subset A d S_{5}$ [10, 11]. The salient feature of all these configurations is that the square of the radius of the D-brane is proportional to an angular momentum $p$ associated with a $S^{1} \subset S^{5}$ rigid motion of the brane. This property marks an important distinction between sphere and AdS giant gravitons: the angular momentum of sphere giants has an upper bound. On the other hand, giants expanded in AdS have no bound on their angular momentum. In 112, 13, 14, 15, BPS dual operators to the above giant gravitons were proposed. Quite interestingly, some non-BPS perturbations of these operators were interpreted as open strings attached to giant gravitons in [18, 19, 20, 21, 22, 23, 24, 25] ${ }^{1}$. Until recently, the anomalous dimensions of these non-BPS operators were not studied as extensively as for single trace BMN operators discussed in [6]. A computational difficulty being that non-BPS operators dual to excited giant gravitons have a number of fields $p$, which taken of order $N$, leads to the failure of the planar approximation. Nevertheless, it is possible to work out the combinatorics and extract the leading order result in the large $N$ limit $^{2}$.

For sphere giant gravitons of maximal size, the resulting boundary conditions for the one-loop anomalous dimension mixing matrix lead to an integrable open spin chain [20]. Computations at two-loop order have recently shown inconsistency with the Bethe Ansatz [23, 24]. So, if the system remains integrable at higher loops, its integrability will not be implemented by a Bethe Ansatz. For non-maximal sphere giants, already at one-loop order, the Hamiltonian is not solvable by a Bethe Ansatz. However, and despite the presence of continuous bands in its spectrum, the Hamiltonian seems to be integrable [25].

In this work we consider dual operators to open strings attached to AdS giant

\footnotetext{
${ }^{1}$ Open strings can also be attached to defect (non-compact) D-branes. In the dCFT, matter fields serve as boundaries for the spin chain and define an integrable open spin chain Hamiltonian [16, 17.

${ }^{2}$ Large $N$ and planar limit are usually taken as synonymous in the literature.
} 
gravitons. The gauge theory description of strings spinning along the AdS directions of the giant requires to consider the action of covariant derivatives on the set of scalar operators. This AdS spinning provides a new parameter $L$ which will be crucial for implementing the BMN scaling. We will deal with operators in a noncompact $\operatorname{sl}(2)$ sub-sector of the superconformal group. To study the anomalous dimensions spectrum of the set we will use the complete one-loop dilation operator of $\mathcal{N}=4 \mathrm{SYM}$, constructed by Beisert in [26], and restrict it to the $\operatorname{sl}(2)$ subsector. In the present work we will not be concerned on the integrability of the Hamiltonian giving the one-loop anomalous dimensions. Instead, we will focus our attention to explore a BMN limit on both dual descriptions of the system. The paper is organized as follow. In section 2 we derive the large $N$ one-loop mixing matrix of anomalous dimensions for the set of operators dual to open strings attached to AdS giant gravitons. Due to the variability in the number of sites in the standard spin chain mapping, we introduce an alternative and more convenient labeling for the operators as states of a bosonic lattice. We provide a semiclassical sigma-model action governing the dynamics of the bosonic Hamiltonian with a large number of sites and also show evidence of the existence of continuous bands in the spectrum of the bosonic Hamiltonian. In section 3 we briefly present the geometrical description of open strings on AdS giants and guided by the AdS/CFT correspondence we see how to confer a geometrical interpretation on the results of section 2. In section 1 we summarize and discuss the results of the paper. Two appendices $\mathrm{A}$ and $\mathrm{B}$ are devoted to the relevant combinatorics for the computations of the section 2 , and the Penrose limit considered in section 3.

\section{Anomalous dimensions for the duals of open strings on AdS giants}

$\mathcal{N}=4$ SYM operators constructed from a single complex scalar field $Z$ in the adjoint representation of $U(N)$ are half-BPS protected. Polynomial operators in $Z$ have their R-charge given by the degree of the polynomial and it is possible to establish a dictionary between these gauge theory half-BPS operators and half-BPS objects in the dual string theory [12, 13, 14, 15]. For instance, a Schur polynomial in $Z$, written in the totally symmetric representation of the permutations group $S_{p}$ is identified with an AdS giant graviton. On the other hand, a Schur polynomial written in the totally antisymmetric representation is identified with a sphere giant graviton [12]. Explicitly, the local gauge theory operator representing an AdS giant graviton with $p$ units of angular momentum is proposed to be

$$
\mathcal{O}_{p}=S_{j_{1} \cdots j_{p}}^{i_{1} \cdots i_{p}} Z_{i_{1}}^{j_{1}} \cdots Z_{i_{p}}^{j_{p}}
$$

where $S_{j_{1} \cdots j_{p}}^{i_{1} \cdots i_{p}}$ is a tensor totally symmetric in all its indices. Its definition together with some useful properties are displayed in the appendix $\mathrm{A}$. 
Open strings attached to giant gravitons (spherical D3-branes) give rise to nonBPS excitations. These states are mapped in the gauge theory to operators like (2.1) but with a $Z$ field replaced by a product of SYM fields and their derivatives, which, usually referred to as a word $W$, represents the open string excitation state. Operators representing excited sphere giant gravitons were previously studied in [18, 19, 20, 21, 22, 23, 24, 25]. Extending the analysis to the case of AdS giant graviton operators is particularly interesting since sphere and AdS giants behave quite differently when their angular momentum $p$ is increased: while the angular momentum of a sphere giant is bounded by $N$ [9], there is no upper bound for the angular momentum of AdS giants (see eqn.(3.5)).

Our attention will be focused on operators of the form,

$$
\mathcal{O}_{p}^{W}=S_{j_{1} \cdots j_{p}}^{i_{1} \cdots i_{p}} Z_{i_{1}}^{j_{1}} \cdots Z_{i_{p-1}}^{j_{p-1}} W_{i_{p}}^{j_{p}}
$$

In particular, we will be interested in open strings following almost null trajectories in order to be able to make contact with gauge theory computations via a BMN limit [6]. This requires to consider open strings spinning fast around the AdS giant graviton. In gauge theory language, this AdS motion is represented by the presence of covariant derivatives in the letters of the word $W$. It will be sufficient for our purposes to consider letters constructed out from a single scalar field $Z$ and covariant derivatives, taken in a unique spacetime direction, acting on it. The infinitely many possible letters $\mathcal{D}^{n} Z$ can be shown to transform in the infinite dimensional spin $j=-\frac{1}{2}$ representation of a non-compact $s l(2)$ subalgebra of the conformal subalgebra. We will study AdS giant operators belonging to this $s l(2)$ sub-sector, which can be shown to be exactly closed [26]. Therefore, the words $W$ we will be considering in (2.2) take the form

$$
W=Z^{\left(n_{1}\right)} \cdot Z^{\left(n_{2}\right)} \cdots Z^{\left(n_{J}\right)}
$$

where,

$$
Z^{(n)}=\frac{1}{n !} \mathcal{D}^{n} Z, \quad \mathcal{D}=\mathcal{D}_{1}+i \mathcal{D}_{2}
$$

Here $\mathcal{D}_{1}$ and $\mathcal{D}_{2}$ are covariant derivatives and the product in (2.3) should be understood as matrix multiplication. Our aim is to study the anomalous dimensions spectrum of the set of operators (2.2) which is conjectured by AdS/CFT to coincide with the open string excitation spectrum of the giant graviton. We will calculate the mixing matrix of anomalous dimension at the large $N$ one-loop approximation.

The complete one-loop dilatation operator $D$ of $\mathcal{N}=4 \mathrm{SYM}$ is known [26]. We will study its restriction to the AdS giant operators (2.2)-(2.3),

$$
D=D_{0}+\frac{g_{\mathrm{YM}}^{2} N}{8 \pi^{2}} D_{1}+\mathcal{O}\left(g_{Y M}^{4}\right)
$$


In (2.5), $D_{0}$ gives the classical dimension of the operator,

$$
D_{0}=\sum_{a=0}^{\infty}(a+1) \operatorname{tr} Z^{(a)} \check{Z}_{(a)}, \quad \text { with } \quad\left(\check{Z}_{(a)}\right)_{j}^{i}=\frac{\delta}{\delta\left(Z^{(a)}\right)_{i}^{j}} .
$$

The one-loop contribution $D_{1}$ in (2.5) is written as

$$
\begin{aligned}
D_{1}= & N^{-1} C_{a b}^{c d}: \operatorname{tr}\left[Z^{(a)}, \check{Z}_{(c)}\right]\left[Z^{(b)}, \check{Z}_{(d)}\right]: \\
= & N^{-1}\left(C_{a b}^{c d}+C_{a b}^{d c}\right):\left(Z^{(a)}\right)_{j}^{i}\left(\check{Z}_{(c)}\right)_{k}^{j}\left(Z^{(b)}\right)_{l}^{k}\left(\check{Z}_{(d)}\right)_{i}^{l}: \\
& -N^{-1}\left(C_{a b}^{c d}+C_{b a}^{d c}\right):\left(Z^{(a)}\right)_{j}^{i}\left(\check{Z}_{(c)}\right)_{k}^{j}\left(\check{Z}_{(d)}\right)_{l}^{k}\left(Z^{(b)}\right)_{i}^{l}:,
\end{aligned}
$$

where sums over repeated indices have been omitted and colons indicate that the variations $\check{Z}$ do not contract on letters within the same colons. The coefficients $C_{a b}^{c d}$ can be obtained from the complete one-loop dilatation operator when its action is restricted to the $\operatorname{sl}(2)$ sub-sector. From eqn. (3.14) of [26] one gets

$$
\begin{aligned}
\left(C_{a b}^{a b}+C_{b a}^{b a}\right) & =-h(a)-h(b), \\
\left(C_{a+n b-m}^{a b}+C_{b-m a+n}^{b a}\right) & =\frac{\delta_{n m}}{|n|}, \quad n=-a, \ldots, b \neq 0,
\end{aligned}
$$

where the harmonic numbers $h(a)$ are defined as,

$$
h(a)=\sum_{i=1}^{a} \frac{1}{i}, \quad h(0)=0 .
$$

We will now analyze the mixing of the operators (2.2)-(2.4) under the action of $D_{1}$. It will be helpful to label the operators we will be working with as,

$$
\mathcal{S}\left(p ; a_{1}, \cdots a_{J}\right):=S_{j_{1} \cdots j_{p}}^{i_{1} \cdots i_{p}} Z_{i_{1}}^{j_{1}} \cdots Z_{i_{p-1}}^{j_{p-1}}\left(Z^{\left(a_{1}\right)} \cdots Z^{\left(a_{J}\right)}\right)_{i_{p}}^{j_{p}} .
$$

The rank of the totally symmetric tensor $p$ is associated with the angular momentum of the giant along a $S^{1} \subset S^{5}$. The number of letters $J$ is dual to the angular momentum of the open string excitation along the same $S^{1}$ as for $p$, and the total number of covariant derivatives $L$ distributed in the word is dual to the open string excitation's angular momentum along a $S^{1} \subset A d S_{5}$ direction. Thus, the integers $a_{n}$ are subject to

$$
\sum_{n=1}^{J} a_{n}=L
$$

It is necessary to normalize the operators (2.10) so that in the large $N$ limit, their free correlation functions are of order one. We define,

$$
\tilde{\mathcal{S}}\left(p ; a_{1}, \cdots a_{J}\right):=\sqrt{\frac{(N+1) !}{(N+p-1) ! p !(p-1) ! N^{J+1}}} \mathcal{S}\left(p ; a_{1}, \cdots a_{J}\right) .
$$


We will exclude the possibility of $a_{1}$ or $a_{J}$ being zero, since in those cases the identity, $S_{j_{1} \cdots j_{p}}^{i_{1} \cdots i_{p}} Z_{i_{1}}^{j_{1}} \cdots Z_{i_{p-1}}^{j_{p-1}}(Z W)_{i_{p}}^{j_{p}}=\frac{1}{p} S_{j_{1} \cdots j_{p+1}}^{i_{1} \cdots i_{p+1}} Z_{i_{1}}^{j_{1}} \cdots Z_{i_{p}}^{j_{p}} W_{i_{p+1}}^{j_{p+1}}-\frac{1}{p} S_{j_{1} \cdots j_{p}}^{i_{1} \cdots i_{p}} Z_{i_{1}}^{j_{1}} \cdots Z_{i_{p}}^{j_{p}} \operatorname{tr}(W)$

allows to write an operator with a $Z$ at the extreme of a word, the lhs, in terms of: a bigger giant with a shorter string (first term, already accounted in the set), and an unexcited D-brane plus a closed string (second term). When computing the mixing among operators (see below), the proper account of the normalization factors for operators shows that the last term in (2.13) is suppressed by a factor $1 / \sqrt{p}$. Since while taking the large $N$ limit we are taking $p \sim N$, the contribution of the last term in (2.13) is irrelevant to the computations.

The action of $D_{1}$ on operators $\tilde{\mathcal{S}}\left(p ; a_{1}, \cdots a_{J}\right)$ is determined by the two derivatives $\check{Z}$ present in (2.7). The possibilities are: (I) both derivatives act on letters of the word $W$ or (II) one derivative acts on a letter of the word and the other on one of the $Z$ fields contracted with the symmetric tensor ${ }^{3}$.

(I) A straightforward computation shows that the leading order contribution to $D_{1}$ in the large $N$ limit comes from the case where both derivatives act on consecutive ${ }^{4}$ letters of the word $W$. The result is

$$
\begin{aligned}
D_{1}^{(I)} \mathcal{S}\left(p ; a_{1}, \cdots a_{J}\right)= & -\left(C_{a b}^{a_{1} a_{2}}+C_{b a}^{a_{2} a_{1}}\right) \mathcal{S}\left(p ; a, b, a_{3}, \cdots a_{J}\right) \\
& -\left(C_{a b}^{a_{2} a_{3}}+C_{b a}^{a_{3} a_{2}}\right) \mathcal{S}\left(p ; a_{1}, a, b, \cdots a_{J}\right)-\cdots \\
& -\left(C_{a b}^{a_{J-1} a_{J}}+C_{b a}^{a_{J} a_{J-1}}\right) \mathcal{S}\left(p ; a_{1}, \cdots a_{J-2}, a, b\right)+\mathcal{O}\left(\frac{1}{N}\right)
\end{aligned}
$$

Using the identity (2.13) we rewrite apart the cases $a=0$ in the first line and $b=0$ in the last line. Taking into account the normalization of the operators (2.12) we obtain,

$$
\begin{aligned}
D_{1}^{(I)} \tilde{\mathcal{S}}\left(p ; a_{1}, \cdots a_{J}\right)= & -\left(C_{a^{\prime} b}^{a_{1} a_{2}}+C_{b a^{\prime}}^{a_{2} a_{1}}\right) \tilde{\mathcal{S}}\left(p ; a^{\prime}, b, a_{3}, \cdots a_{J}\right) \\
& -\left(C_{a b}^{a_{2} a_{3}}+C_{b a}^{a_{3} a_{2}}\right) \tilde{\mathcal{S}}\left(p ; a_{1}, a, b, \cdots a_{J}\right)-\cdots \\
& -\left(C_{a b^{\prime}}^{a_{J-1} a_{J}}+C_{b^{\prime} a}^{a_{J} a_{J-1}}\right) \tilde{\mathcal{S}}\left(p ; a_{1}, \cdots a_{J-2}, a, b^{\prime}\right) \\
& -\sqrt{1+\frac{p}{N}}\left(C_{0 b}^{a_{1} a_{2}}+C_{b 0}^{a_{2} a_{1}}\right) \tilde{\mathcal{S}}\left(p+1 ; b, a_{3}, \cdots a_{J}\right) \\
& -\sqrt{1+\frac{p}{N}}\left(C_{a 0}^{a_{J-1} a_{J}}+C_{0 a}^{a_{J} a_{J-1}}\right) \tilde{\mathcal{S}}\left(p+1 ; a_{1}, \cdots a_{J-2}, a\right) \\
& +\mathcal{O}\left(\frac{1}{\sqrt{p}}\right)
\end{aligned}
$$

\footnotetext{
${ }^{3}$ When both derivatives act on $Z$ fields contracted with the symmetric tensor, the contribution is proportional to $C_{00}^{00}$ which is zero (cf. 2.8)).

${ }^{4}$ Contributions from the action of $\check{Z}$ on non-consecutive letters are sub-leading.
} 
Here a primed repeated index indicates that its summation excludes the value zero. The last two terms in (2.15) show that the dilatation operator mixes states with words of different lengths. This instance is similar to that of non-maximal sphere giant gravitons [21, 25] and the mixing between operators with words of different lengths could have been expected. The variation in the number of letters of the word $W$ can be pictured, from the string point of view, as coming from the exchange of the angular momentum along the $S^{1} \subset S^{5}$ between the string and the giant. The open string gets dragged by the movement of the giant graviton while propagating. Notice also that the factor describing the mixing of words of different lengths is $\sqrt{1+p / N}$. The sign inside the square root has changed with respect to the similar factor appearing in the sphere giant case. This last fact reflects that $p$ can increase arbitrarily for AdS giants.

(II) The leading order result, considering as before $p \sim N$, is

$$
\begin{aligned}
D_{1}^{(I I)} \tilde{\mathcal{S}}\left(p ; a_{1}, \cdots a_{J}\right)= & -\left(1+\frac{p}{N}\right)\left(C_{b 0}^{a_{1} 0}+C_{0 b}^{0 a_{1}}\right) \tilde{\mathcal{S}}\left(p ; b, a_{2}, \cdots a_{J}\right) \\
& -\left(1+\frac{p}{N}\right)\left(C_{b 0}^{a_{J} 0}+C_{0 b}^{0 a_{J}}\right) \tilde{\mathcal{S}}\left(p ; a_{1}, \cdots a_{J-1}, b\right) \\
& -\sqrt{1+\frac{p}{N}}\left(C_{a^{\prime} b}^{0 a_{1}}+C_{b a^{\prime}}^{a_{1} 0}\right) \tilde{\mathcal{S}}\left(p-1 ; a^{\prime}, b, a_{2}, \cdots a_{J}\right) \\
& -\sqrt{1+\frac{p}{N}}\left(C_{a b^{\prime}}^{a_{J} 0}+C_{b^{\prime} a^{\prime}}^{0 a_{J}}\right) \tilde{\mathcal{S}}\left(p-1 ; a_{1}, \cdots a_{J-1}, a, b^{\prime}\right) \\
& -\frac{p}{N}\left(C_{0 b}^{0 a_{1}}+C_{b 0}^{a_{1} 0}+C_{0 b}^{a_{1} 0}+C_{b 0}^{0 a_{1}}\right) \tilde{\mathcal{S}}\left(p ; b, a_{2}, \cdots a_{J}\right) \\
& -\frac{p}{N}\left(C_{0 b}^{0 a_{J}}+C_{b 0}^{a_{J} 0}+C_{0 b}^{a_{1} 0}+C_{b 0}^{0 a_{1}}\right) \tilde{\mathcal{S}}\left(p ; a_{1}, \cdots a_{J-1}, b\right) \\
& +\mathcal{O}\left(\frac{1}{\sqrt{p}}\right) .
\end{aligned}
$$

Defining the parameter

$$
\alpha \equiv \sqrt{1+\frac{p}{N}} .
$$


and using the coefficients (2.8), the result (2.15) can be rephrased as

$$
\begin{aligned}
D_{1}^{(I)} \tilde{\mathcal{S}}\left(p ; a_{1}, \cdots a_{J}\right)= & \left(h\left(a_{1}\right)+h\left(a_{2}\right)\right) \tilde{\mathcal{S}}\left(p ; a_{1}, \cdots a_{J}\right) \\
& -\sum_{n=-a_{2}}^{a_{1}-1} \frac{1}{|n|} \tilde{\mathcal{S}}\left(p ; a_{1}-n, a_{2}+n,, \cdots a_{J}\right) \\
& +\left(h\left(a_{2}\right)+h\left(a_{3}\right)\right) \tilde{\mathcal{S}}\left(p ; a_{1}, \cdots a_{J}\right) \\
& -\sum_{n=-a_{3}}^{a_{2}} \frac{1}{|n|} \tilde{\mathcal{S}}\left(p ; a_{1}, a_{2}-n, a_{3}+n, \cdots a_{J}\right)-\cdots \\
& +\left(h\left(a_{J-1}\right)+h\left(a_{J}\right)\right) \tilde{\mathcal{S}}\left(p ; a_{1}, \cdots, a_{J}\right) \\
& -\sum_{J-1}-1 \\
& -\frac{\alpha}{a_{1}} \tilde{\mathcal{S}}\left(p+1 ; a_{1}+a_{2}, a_{3}, \cdots a_{J}\right) \\
& -\frac{\alpha}{a_{J}} \tilde{\mathcal{S}}\left(p+a_{1}, \cdots, a_{J-1}-n, a_{J}+n\right)
\end{aligned}
$$

The first six lines can be identified with the action of an open $\operatorname{sl}(2)$ spin chain Hamiltonian under the standard identification Word $\leftrightarrow$ Spin Chain State [7, 8, 26]. The last two lines indicate that sites can be annihilated at the boundaries of the chain. Similarly, (2.16) can be rewritten as,

$$
\begin{aligned}
D_{1}^{(I I)} \tilde{\mathcal{S}}\left(p ; a_{1}, \cdots a_{J}\right)= & \alpha^{2}\left(h\left(a_{1}\right)+h\left(a_{J}\right)\right) \tilde{\mathcal{S}}\left(p ; a_{1}, \cdots a_{J}\right) \\
& -\alpha \sum_{n=1}^{a_{1}} \frac{1}{n} \tilde{\mathcal{S}}\left(p-1 ; n, a_{1}-n, a_{2}, \cdots a_{J}\right) \\
& -\alpha \sum_{n=1}^{a_{J}} \frac{1}{n} \tilde{\mathcal{S}}\left(p-1 ; a_{1}, \cdots, a_{J-1}, a_{J}-n, n\right) \\
& -\left(\alpha^{2}-1\right)\left(h\left(a_{1}-1\right)+h\left(a_{J}-1\right)\right) \tilde{\mathcal{S}}\left(p ; a_{1}, \cdots a_{J}\right) \\
& +\mathcal{O}\left(\frac{1}{\sqrt{p}}\right) .
\end{aligned}
$$

The first and last lines represent the action of identity terms, while the two middle ones show that sites can be created at the boundaries of the chain.

Describing different words as open $s l(2)$ spin chain states is not the most convenient picture. Since sites can be created or annihilated at the boundaries, one would have to deal with spin chains of variable length. In order to find a more appropriate labeling of the words set, it is crucial to note that the total number of covariant derivatives in the word $W$ is conserved under the action of the 1-loop dilatation operator. We choose then to label the words (2.3) by stating the number of $Z$ fields between consecutive covariant derivatives. Consider, for instance, the word

$$
Z^{(1)} Z^{(0)} Z^{(2)} Z^{(1)} \sim \mathcal{D} Z Z \mathcal{D D} Z \mathcal{D} Z
$$


We specify it by indicating that there are two $Z$ s between the first and the second derivative $\mathcal{D}$, no one between the second and the third and one $Z$ between the third and the fourth derivative. A word with $L+1$ covariant derivatives will then be labeled as a bosonic state of a lattice with $L$ sites,

$$
\mathcal{D} Z^{n_{1}} \mathcal{D} Z^{n_{2}} \mathcal{D} \cdots \mathcal{D} Z^{n_{L}} \mathcal{D} Z \quad \leftrightarrow \quad\left|n_{1}, n_{2}, \cdots, n_{L}\right\rangle
$$

with $n_{i}=0,1, \ldots$. The variability of the $s l(2)$ spin chain length is translated, in the labeling (2.21), into a variability of the total occupation number of the lattice. This variability will take place at the size of the giant graviton expense. However, since the probability of a $Z$ entering or leaving the word is the same, we expect that $p$ can be consistently taken as a constant. A posteriori we will check that occupation number of $Z \mathrm{~s}$ for the ground state is much smaller than $p$. Summarizing, the total number of bosons in the bosonic lattice is equal to the total number of $Z \mathrm{~s}$ in the word.

To translate the action of $D_{1}(2.18)-(2.19)$ to the bosonic language $(2.21)$, we introduce shift operators $\hat{a}_{i}^{\dagger}$ and $\hat{a}_{i}$ that rise and lower the occupation number of $i^{\text {th }}$ site

$$
\hat{a}_{i}^{\dagger}\left|n_{i}\right\rangle=\left|n_{i}+1\right\rangle, \quad \hat{a}_{i}\left|n_{i}\right\rangle=\left|n_{i}-1\right\rangle .
$$

Note that their action does not involve the square roots of the standard oscillator-like operators, therefore,

$$
\hat{a}_{i} \hat{a}_{i}^{\dagger}=I, \quad \hat{a}_{i}^{\dagger} \hat{a}_{i}=I-P_{i}^{0} \equiv I-|0\rangle\left\langle\left. 0\right|_{i} .\right.
$$

Consider the word that begins as

$$
Z^{\left(a_{1}\right)} Z^{\left(a_{2}\right)} \ldots \sim \overbrace{\mathcal{D} \cdots \mathcal{D}}^{a_{1}} Z \overbrace{\mathcal{D} \cdots \mathcal{D}}^{a_{2}} Z \cdots
$$

The only restriction we have is that $a_{1} \neq 0$ (cf. (2.13)). The first $a_{1}-1$ sites are empty states in the bosonic language and the $a_{1}^{\text {th }}$ is necessarily occupied. The amount of bosons occupying it depends on the subsequent $a_{i}$,

$$
Z^{\left(a_{1}\right)} Z^{\left(a_{2}\right)} \cdots \leftrightarrow\left\{\begin{array}{c}
|\overbrace{0, \cdots, 0}^{a_{1}-1}, 1, \cdots\rangle \quad \text { if } a_{2} \neq 0 \\
|\overbrace{0, \cdots, 0}^{a_{1}-1}, \cdots\rangle \quad \text { if } a_{2}=0 \wedge a_{3} \neq 0 \\
\vdots \\
\vdots
\end{array}\right.
$$

Let us consider in detail the translation of some of the terms in (2.18) and finally present the complete result. We can think of $D_{1}$ as the Hamiltonian of a bosonic 
lattice. Defining $H=\lambda D_{1}$, the diagonal terms in (2.18) can be understood as amounts of energy for each site occupied. Their total contribution is

$$
E_{\text {osc }} \sim \lambda\left(h\left(a_{1}\right)+2 h\left(a_{2}\right)+\cdots+2 h\left(a_{J-1}\right)+h\left(a_{J}\right)\right),
$$

where we have introduced the 't Hooft parameter $\lambda=\frac{g_{Y M}^{2} N}{8 \pi^{2}}$. This total contribution can be obtained in the following way: for each site of the lattice there is no contribution if it is empty, if the site is occupied the amount is independent of the number of bosons in the site ${ }^{5}$. However, the amount for each occupied site depends on the occupancy of their neighbors. The contribution in question is $h\left(e_{L}+1\right)+h\left(e_{R}+1\right)$, where $e_{L}$ is the number of consecutive empty sites (if any) to the left and $e_{R}$ is the number of consecutive empty sites (if any) to the right. These diagonal terms of the lattice Hamiltonian are written as

$$
\begin{aligned}
H_{\text {osc }}= & \lambda \sum_{m=1}^{L-1} \sum_{l=1}^{L-m} \frac{1}{m}\left(\hat{a}_{l}^{\dagger} \hat{a}_{l}+\hat{a}_{l+m}^{\dagger} \hat{a}_{l+m}\right)\left(\prod_{s=l+1}^{l+m-1} P_{s}^{0}\right) \\
& +\lambda \sum_{l=1}^{L} \frac{1}{l} \hat{a}_{l}^{\dagger} \hat{a}_{l}\left(\prod_{s=1}^{l-1} P_{s}^{0}\right)+\sum_{l=1}^{L} \frac{1}{L+1-l} \hat{a}_{l}^{\dagger} \hat{a}_{l}\left(\prod_{s=l+1}^{L} P_{s}^{0}\right) .
\end{aligned}
$$

There are also terms in (2.18) that represent the exchange of $n$ covariant derivatives between consecutive letters of the word. In the bosonic labeling, they are seen as hopping terms. Since more than one covariant derivative can be exchanged, the hopping is not only between nearest neighbors. A boson can be exchanged between non-nearest neighbor sites as long as all the sites between them are empty. These hopping terms can be written as,

$$
H_{\text {hopping }}=-\lambda \sum_{m=1}^{L-1} \sum_{l=1}^{L-m} \frac{1}{m}\left(\hat{a}_{l+m}^{\dagger} \hat{a}_{l}+\hat{a}_{l}^{\dagger} \hat{a}_{l+m}\right)\left(\prod_{s=l+1}^{l+m-1} P_{s}^{0}\right) .
$$

A similar analysis can be repeated to rephrase the terms corresponding to the creation and annihilation of letters at the boundaries as well as the diagonal terms in (2.19). Altogether, the action of $D_{1}$ on the operators corresponding to open strings on AdS giant gravitons, is given by the action of the following bosonic lattice Hamiltonian,

$$
\begin{aligned}
H= & \lambda \sum_{m=1}^{L-1} \sum_{l=1}^{L-m} \frac{1}{m}\left(\hat{a}_{l}^{\dagger}-\hat{a}_{l+m}^{\dagger}\right)\left(\hat{a}_{l}-\hat{a}_{l+m}\right)\left(\prod_{s=l+1}^{l+m-1} P_{s}^{0}\right) \\
& +\lambda \sum_{l=1}^{L} \frac{1}{l}\left(\hat{a}_{l}^{\dagger} \hat{a}_{l}+\alpha^{2}-\alpha\left(\hat{a}_{l}+\hat{a}_{l}^{\dagger}\right)+\left(1-\alpha^{2}\right) P_{l}^{0}\right)\left(\prod_{s=1}^{l-1} P_{s}^{0}\right) \\
& +\lambda \sum_{l=1}^{L} \frac{1}{L+1-l}\left(\hat{a}_{l}^{\dagger} \hat{a}_{l}+\alpha^{2}-\alpha\left(\hat{a}_{l}+\hat{a}_{l}^{\dagger}\right)+\left(1-\alpha^{2}\right) P_{l}^{0}\right)\left(\prod_{s=l+1}^{L} P_{s}^{0}\right),
\end{aligned}
$$

\footnotetext{
${ }^{5}$ This is the reason for having chosen the shift operators $(2.22)$ instead of ordinary creation and annihilation operators
} 
The terms proportional to $\alpha$ in the second and third lines of (2.29) represent sinks and sources for bosons. Bosons can be created or annihilated at the $l^{\text {th }}$ site of the lattice, as long as all sites in between the $l^{\text {th }}$ site and one of the boundaries are empty. As a consequence, the total number of bosons does not commute with the Hamiltonian. This represents a serious difficulty in trying to diagonalize (2.29): their eigenvalues will not have a definite number of bosons. Nevertheless, it is possible to build a uniform coherent eigenstate with zero eigenvalue. This is nothing but the ground state of (2.29),

$$
\left|\Psi_{0}\right\rangle=\left|\alpha^{-1}, \ldots, \alpha^{-1}\right\rangle=\left(\frac{\alpha^{2}-1}{\alpha^{2}}\right)^{L / 2} \sum_{n_{1}, \ldots, n_{L}=0}^{\infty} \alpha^{-\left(n_{1}+\cdots+n_{L}\right)}\left|n_{1}, \ldots, n_{L}\right\rangle .
$$

However, we do not know how to solve the eigenvalue problem in general.

\subsection{The Semiclassical Limit}

We will show in this section that the dynamics, in the $L \rightarrow \infty$ limit, given by the Hamiltonian (2.29) is governed by a semiclassical sigma-model action. The action will be obtained by taking a continuum limit of the path integral representation of the evolution operator written in a coherent states basis. It was shown in 27] that the semiclassical action obtained for the Heisenberg spin chain Hamiltonian, which describes the planar 1-loop anomalous dimension of single trace operators in a $s u(2)$ sub-sector, can be related to the closed string action when appropriate gauge choice and specific limits are taken. A similar identification was done for single trace operators in a $\operatorname{sl}(2)$ sub-sector in [28, 29, 30].

The semiclassical action for the dynamics in the $L \rightarrow \infty$ limit reads,

$$
S=\int d t\left(i\left\langle z_{1} \ldots z_{L}\left|\frac{d}{d t}\right| z_{1} \ldots z_{L}\right\rangle-\left\langle z_{1} \ldots z_{L}|H| z_{1} \ldots z_{L}\right\rangle\right),
$$

We are dealing with a bosonic Hamiltonian and the coherent states we use are defined to be eigenstates of the shift operator $\hat{a}$,

$$
|z\rangle=\sqrt{1-|z|^{2}} \sum_{n=0}^{\infty} z^{n}|n\rangle, \quad \text { with } \quad|z|<1,
$$

As known, coherent states constitute a non-orthogonal and overcomplete basis. The overlapping between states is

$$
\left\langle z \mid z^{\prime}\right\rangle=\frac{\sqrt{1-|z|^{2}} \sqrt{1-\left|z^{\prime}\right|^{2}}}{1-\bar{z} z^{\prime}} .
$$

We parameterize the coherent states as $z_{l}(t)=u_{l}(t) e^{i \phi_{l}(t)}$. The first term in the integrand o (2.31) takes then the form

$$
i\left\langle z_{1} \ldots z_{L}\left|\frac{d}{d t}\right| z_{1} \ldots z_{L}\right\rangle=-\sum_{l=1}^{L} \frac{u_{l}^{2} \dot{\phi}_{l}}{1-u_{l}^{2}} .
$$


In the limit of large $L$, the functions $u_{l}(t)$ and $\phi_{l}(t)$ can be considered to be continuous functions $u(t, \sigma)$ and $\phi(t, \sigma)$ with $0 \leq \sigma \leq 1$, the sum over $l$ being converted into an integral over $\sigma$,

$$
i\left\langle z_{1} \ldots z_{L}\left|\frac{d}{d t}\right| z_{1} \ldots z_{L}\right\rangle=-L \int_{0}^{1} d \sigma \frac{u^{2} \dot{\phi}}{1-u^{2}}
$$

Here a dot denotes a derivative with respect to $t$. For the second term in the integrand of (2.31) one obtains

$$
\begin{aligned}
\langle H\rangle= & \left\langle z_{1} \ldots z_{L}|H| z_{1} \ldots z_{L}\right\rangle \\
= & \lambda \sum_{m=1}^{L-1} \sum_{l=1}^{L-m} \frac{1}{m}\left(u_{l+m}-u_{l}\right)^{2}\left(\prod_{s=l+1}^{l+m-1}\left(1-u_{s}^{2}\right)\right) \\
& -\lambda \sum_{m=1}^{L-1} \sum_{l=1}^{L-m} \frac{1}{m} 2 u_{l} u_{l+m}\left(\cos \left(\phi_{l+m}-\phi_{l}\right)-1\right)\left(\prod_{s=l+1}^{l+m-1}\left(1-u_{s}^{2}\right)\right) \\
& +\lambda \sum_{m=1}^{L} \frac{1}{m}\left(\alpha^{2} u_{m}^{2}+1-2 \alpha u_{m} \cos \phi_{m}\right)\left(\prod_{s=1}^{m-1}\left(1-u_{s}^{2}\right)\right) \\
& +\lambda \sum_{m=1}^{L} \frac{1}{L+1-m}\left(\alpha^{2} u_{m}^{2}+1-2 \alpha u_{m} \cos \phi_{m}\right)\left(\prod_{s=m+1}^{L}\left(1-u_{s}^{2}\right)\right) .
\end{aligned}
$$

In the large $L$ limit of (2.36), sums over $l$ (in the first two lines) are going to be approximated by integrals over a continuous variable $\sigma$, while sums over $m$ are going to be approximated by geometric sums. Identifying $f_{l} \simeq f(\sigma)$,

$$
f_{l+m} \simeq f(\sigma)+\frac{m}{L} f^{\prime}(\sigma)+\frac{1}{2}\left(\frac{m}{L}\right)^{2} f^{\prime \prime}(\sigma)+\mathcal{O}\left(m^{3} / L^{3}\right)
$$

Approximating the sums as indicated, the contributions to 2.36 can be gathered 
order by order in powers of $1 / L$. The result is

$$
\begin{aligned}
\langle H\rangle= & -\frac{\lambda}{L} \int_{0}^{1} d \sigma \frac{1}{u^{4}}\left(u^{\prime 2}+u^{2} \phi^{\prime 2}\right) \\
& +\left.\lambda \sum_{m=1}^{L} \frac{\left(1-u^{2}\right)^{m-1}}{m}\left(\alpha^{2} u^{2}+1-2 \alpha u \cos \phi\right)\right|_{\sigma=0} \\
& +\frac{\lambda}{L} \sum_{m=1}^{L} \frac{m-1}{m}\left(1-u^{2}\right)^{m-1}\left(2 u^{\prime}(u-\alpha \cos \phi)+2 \alpha u \sin \phi \phi^{\prime}\right. \\
& \left.-(m-2) \frac{u u^{\prime}}{1-u^{2}}\left(u^{2}+\alpha^{2}-2 \alpha u \cos \phi\right)-m\left(1-\alpha^{2}\right) u u^{\prime}\right)\left.\right|_{\sigma=0} \\
& +\left.\lambda \sum_{m=1}^{L} \frac{\left(1-u^{2}\right)^{m-1}}{m}\left(\alpha^{2} u^{2}+1-2 \alpha u \cos \phi\right)\right|_{\sigma=1} \\
& +\frac{\lambda}{L} \sum_{m=1}^{L} \frac{m-1}{m}\left(1-u^{2}\right)^{m-1}\left(2 u^{\prime}(u-\alpha \cos \phi)+2 \alpha u \sin \phi \phi^{\prime}\right. \\
& \left.-(m-2) \frac{u u^{\prime}}{1-u^{2}}\left(u^{2}+\alpha^{2}-2 \alpha u \cos \phi\right)-m\left(1-\alpha^{2}\right) u u^{\prime}\right)\left.\right|_{\sigma=1}+\mathcal{O}\left(1 / L^{2}\right) .
\end{aligned}
$$

Primes denote derivatives with respect to $\sigma$. The bulk term of this semiclassical Hamiltonian is of order $\lambda / L$. However, the boundary terms leading order is $\lambda$. Then, the semiclassical configurations with the lowest anomalous dimension are going to be those satisfying the following Dirichlet boundary conditions,

$$
\left.\left(\alpha^{2} u^{2}+1-2 \alpha u \cos \phi\right)\right|_{\sigma=0,1}=0 \text {. }
$$

This is equivalent to require,

$$
\begin{aligned}
& \left.u\right|_{\sigma=0,1}=\frac{1}{\alpha} \\
& \left.\phi\right|_{\sigma=0,1}=0 .
\end{aligned}
$$

Note that imposing the boundary conditions (2.40) cancels not only the boundary terms of order $\lambda$ but also those of order $\lambda / L$. Finally, the semiclassical action for the large $L$ limit with boundary conditions (2.40) is,

$$
S=-L \int d t \int_{0}^{1} d \sigma\left(\frac{u^{2} \dot{\phi}}{1-u^{2}}-\frac{\lambda}{L^{2} u^{4}}\left(u^{\prime 2}+u^{2} \phi^{\prime 2}\right)\right) .
$$

At the end of section 3.1, we will give a geometrical interpretation of the coherent states parameters $u$ and $\phi$. To this end, it is convenient to introduce the variable $r \equiv 1 / u$, taking values $r \in(1, \infty)$. The action (2.41) is rewritten as

$$
S=-L \int d t \int_{0}^{1} d \sigma\left(\frac{\dot{\phi}}{r^{2}-1}-\frac{\lambda}{L^{2}}\left(r^{\prime 2}+r^{2} \phi^{\prime 2}\right)\right)
$$


and the Dirichlet boundary conditions (2.40) as,

$$
\begin{aligned}
& \left.r\right|_{\sigma=0,1}=\alpha=\sqrt{1+\frac{p}{N}} \\
& \left.\phi\right|_{\sigma=0,1}=0 .
\end{aligned}
$$

The ground state of this semiclassical action is the constant configuration $r=\alpha$ and $\phi=0$, which is a coherent state $z=1 / \alpha$ uniformly distributed along the lattice. This is precisely the ground state (2.30).

\subsection{Continuous bands}

In this section we argue that the Hamiltonian (2.29) has continuous bands in its spectrum. The variability in the mean occupation number together with these continuous bands indicate that it is possible to construct states whose evolution gives a growing mean occupation number.

Among the family of Hamiltonians (2.29) we are only able to exactly diagonalize the case $L=1$. The one site, the Hamiltonian is,

$$
\begin{aligned}
H & \left.=2 \lambda\left(\hat{a}^{\dagger} \hat{a}+\alpha^{2}-\alpha\left(\hat{a}+\hat{a}^{\dagger}\right)+\left(1-\alpha^{2}\right) P^{0}\right)\right) \\
& =2 \lambda \alpha^{2}\left(\hat{a}^{\dagger} \hat{a}+\frac{1}{\alpha^{2}}-\frac{1}{\alpha}\left(\hat{a}+\hat{a}^{\dagger}\right)\right) .
\end{aligned}
$$

A ground state and a continuum after a gap constitute the whole set of eigenstates,

$$
\begin{aligned}
\left|\Psi_{0}\right\rangle & =\left(\frac{\alpha^{2}-1}{\alpha^{2}}\right)^{1 / 2} \sum_{n=0}^{\infty} \frac{1}{\alpha^{n}}|n\rangle, \\
|\Psi(k)\rangle & =\sum_{n=0}^{\infty}\left(\sin k n-\frac{1}{\alpha} \sin k(n+1)\right)|n\rangle, \quad \text { with } 0 \leq k \leq \pi .
\end{aligned}
$$

The energy of the ground state is zero, while the energy of the states in the band is

$$
E(k)=2 \lambda\left(1-2 \alpha \cos k+\alpha^{2}\right) .
$$

The gap between the ground state and the band is

$$
2 \lambda(\alpha-1)^{2}
$$

States in the band satisfy a delta-function normalization. Normalizable wave-packets can be built out of them, whose mean occupation numbers grow monotonically as evolution takes place.

To analyze the existence of continuous bands for larger $L$ we consider the system semiclassically. As we have already argued, the action (2.31) governs the dynamics of our system in the $L \rightarrow \infty$ limit. The idea we pursue is simple: energies for which 
orbits in phase space are open, correspond to a continuum in the spectrum of the quantized system.

To begin with, notice that conjugate momenta $p_{u_{l}}$ and $p_{\phi_{l}}$ are subject to constraints $^{6}$. Therefore, the phase space coincides with the configuration space $\left(u_{l}, \phi_{l}\right)$, which is a product of $L$ discs of radii $\mathrm{r}=1$. We are interested in the values of the semiclassical Hamiltonian (2.36), which allow open orbits in the phase space. Since the energy is a conserved quantity, orbits are constrained to hypersurfaces of constant energy. Therefore, open orbits are possible for energies, whose hypersurfaces intersect the boundary of the phase space. We are be only interested in the minimal amount of energy that a state would need to reach a continuous band. Then, we have to compute the minimum of each of the functions resulting when one of the $u_{l}$ takes the values 1 . These $L$ functions are bounded from below by polynomials on $u_{l}$

$$
\begin{aligned}
\left.\langle H\rangle\right|_{u_{k}=1} \geq \mathcal{M}_{L}^{k}= & \left.\lambda \sum_{m=1}^{L-1} \sum_{l=1}^{L-m} \frac{1}{m}\left(u_{l+m}-u_{l}\right)^{2}\left(\prod_{s=l+1}^{l+m-1}\left(1-u_{s}^{2}\right)\right)\right|_{u_{k}=1} \\
& +\left.\lambda \sum_{m=1}^{L} \frac{1}{m}\left(\alpha^{2} u_{m}^{2}+1-2 \alpha u_{m}\right)\left(\prod_{s=1}^{m-1}\left(1-u_{s}^{2}\right)\right)\right|_{u_{k}=1} \\
& +\left.\lambda \sum_{m=1}^{L} \frac{1}{L+1-m}\left(\alpha^{2} u_{m}^{2}+1-2 \alpha u_{m}\right)\left(\prod_{s=m+1}^{L}\left(1-u_{s}^{2}\right)\right)\right|_{u_{k}=1} .
\end{aligned}
$$

which are obtained by setting all angles $\phi_{l}$ to zero. The minima of the $\mathcal{M}_{L}^{k}$ then give us the minima of $\left.\langle H\rangle\right|_{u_{k}=1}$. It is straightforward to verify that the stationary point equations are satisfied with the following values,

$$
\begin{array}{ll}
\frac{1}{u_{l}^{*}}=\left(\frac{1-\alpha}{k}\right) l+\alpha & \text { if } l \leq k, \\
\frac{1}{u_{l}^{*}}=\left(\frac{\alpha-1}{(L+1-k)}\right) l+\frac{L+1-k \alpha}{(L+1-k)} & \text { if } l>k .
\end{array}
$$

One can also see that these critical points are minima of each $\mathcal{M}_{L}^{k}$, and the energy evaluated on them is

$$
\left.\langle H\rangle\right|_{u_{l}=u_{l}^{*}, \phi_{l}=0}=\frac{\lambda(L+1)(\alpha-1)^{2}}{k(L+1-k)} .
$$

It is not difficult to see that the intersection where the central site takes the value one gives a minimum of the expression (2.52). Summarizing, the minimal amount of energy for having open orbits is

$$
E_{\text {cont }}=\left\{\begin{array}{cl}
\frac{4 \lambda(\alpha-1)^{2}}{(L+1)} & \text { if } L \text { is odd } \\
\frac{4 \lambda(L+1)(\alpha-1)^{2}}{L(L+2)} & \text { if } L \text { is even } .
\end{array}\right.
$$

\footnotetext{
${ }^{6}$ From (2.34) one gets $p_{u_{l}}=0$ and $p_{\phi_{l}}=\frac{u_{l}^{2}}{u_{l}^{2}-1}$.
} 
This analysis enables us to conclude that in the large $L$ limit, the minimal energy to reach the lowest continuous band in the spectrum is,

$$
E_{\mathrm{cont}}=\frac{4 \lambda(\alpha-1)^{2}}{L}
$$

Operators dual to long semiclassical strings, with energy order $\lambda / L$, could show a growing occupation number. The existence of these configurations indicate that the D-brane might be unstable if it is excited with long strings.

It is possible to give a picture of these configurations from the geometrical viewpoint. The motion of the giant along the $\psi$ direction of the $S^{5}$ exerts centrifugal forces on long strings attached to it. For sufficiently long strings, these forces could excess the string tension. To see if this phenomenon constitutes a D-brane instability, it is necessary to study how the diminution in giant size back-react on these configurations with growing occupation number. However, this analysis is beyond the validity of the approximations we made in the derivation of (2.29).

\section{Open strings on AdS giant gravitons}

Let us briefly state some of the geometrical properties of open strings ending on AdS giant gravitons. We will see that some of them have already appeared as outcomes of the Hamiltonian (2.29).

We write the metric of $A d S_{5} \times S^{5}$ in global coordinates

$$
d s^{2}=R^{2}\left(-\cosh ^{2} \rho d t^{2}+d \rho^{2}+\sinh ^{2} \rho d \Omega_{3}{ }^{2}+d \theta^{2}+\cos ^{2} \theta d \psi^{2}+\sin ^{2} \theta d \Omega_{3}^{\prime 2}\right),
$$

and the 3-sphere metrics as

$$
\begin{aligned}
& d \Omega_{3}^{2}=d \varphi^{2}+\cos ^{2} \varphi d \eta^{2}+\sin ^{2} \varphi d \xi^{2} \\
& d \Omega_{3}^{\prime 2}=d \varphi^{\prime 2}+\cos ^{2} \varphi^{\prime} d \eta^{\prime 2}+\sin ^{2} \varphi^{\prime} d \xi^{\prime 2} .
\end{aligned}
$$

A RR 4-form potential supports the geometry, its self-dual field strength possessing $N$ units of flux on the $S^{5}$. The supergravity equations of motion relate the radius $R$ in (3.1) to the $F_{5}$ flux on $S^{5}$ according to $R^{4}=4 \pi g_{s} N \alpha^{2}$.

Giant gravitons are spinning spheric D3-branes that expand in either the 3spheres $\Omega_{3}^{\prime}$ or $\Omega_{3}$. They spin rigidly along the $\psi$ direction inside the $S^{5}$ and are located either at $\rho=0$ and $\theta=\theta_{0}$, or at $\theta=0$ and $\rho=\rho_{0}$ [9, 10, 11]. The former correspond to D-branes expanded into a $S^{\prime 3} \subset S^{5}$ and we refer to them as sphere giant gravitons. The latter, which we call AdS giant gravitons, correspond to Dbranes expanded into a $S^{3} \subset A d S_{5}$ and the previous section was devoted to study of their gauge theory dual operators. 
We denote by $\left(\tau, \sigma_{1}, \sigma_{2}, \sigma_{3}\right)$ the world-volume coordinates of an AdS giant. The D3-brane equations of motion are solved by choosing the embedding space-time coordinates to be given by

$$
\begin{aligned}
& t=\tau, \quad \rho=\rho_{0}, \quad \theta=0, \quad \psi=\tau \\
& \varphi=\sigma_{1}, \quad \eta=\sigma_{2}, \quad \xi=\sigma_{3} .
\end{aligned}
$$

Independently of the position $\rho_{0}$ of the giant, the angular velocity is $\dot{\psi}=1$. Thus, its center of mass, located at $\rho=0$, moves along a null trajectory. Nevertheless, each element of the giant travels in a time-like orbit.

The radius of the spherical giant gravitons and their angular momentum $p$ along the $\psi$ direction inside the $S^{5}$ are given in terms of the radial AdS coordinate $\rho$ by

$$
\begin{aligned}
& r=R \sinh \rho_{0}, \\
& p=N \sinh ^{2} \rho_{0} .
\end{aligned}
$$

From this equations one gets that $r^{2}=2 \sqrt{\pi g_{s}} \alpha^{\prime} p / \sqrt{N}$. The DBI action, of which giant gravitons are D3-branes solutions, is then a valid approximation if $p>>\sqrt{N}$.

Weakly excited strings with a large angular momentum, i.e. those traveling along almost null trajectories on any spacetime, can be approximated as moving on an effective pp-wave geometry [6]. We will be interested in open strings having large angular momentum along $\psi$, the Penrose limit can be understood as a large $N$ limit, with the angular momentum $p$ growing proportional to $N$. By looking at (3.4) and (3.5) one realizes that the value $\rho_{0}$ must be kept constant and therefore the radius of the giant diverges. The open string will be effectively attached to a flat D3-brane in a pp-wave background ${ }^{7}$.

For definiteness, let us consider a trajectory along $\psi$ and $\eta$, keeping $\varphi=0$. A null trajectory requires

$$
R^{2}\left(-\cosh ^{2} \rho_{0} \dot{t}^{2}+\sinh ^{2} \rho_{0} \dot{\eta}^{2}+\dot{\psi}^{2}\right)=0 .
$$

Since we want the trajectory to be contained in the D-brane world-volume, the parametrization (3.3) $\dot{t}=1$ and $\dot{\psi}=1$ necessarily implies $\dot{\eta}= \pm 1$. This means that the coordinates $t, \psi$ and $\eta$ are equally rated along this null trajectory. This null trajectory is a null geodesic of $A d S_{5} \times S^{5}$ and we use it to take a Penrose limit (see appendix $\mathrm{B}$ ). Looking at the open string spectrum on the pp-wave background (B.16), it is natural to expect for the first excited states of (2.29), the following eigenvalues in the limit of large $L$ but $\lambda\left(\alpha^{2}-1\right)^{2} / L^{2}$ fixed and small,

$$
E_{n} \approx \frac{\lambda \pi^{2}\left(\alpha^{2}-1\right)^{2} n^{2}}{L^{2}}
$$

\footnotetext{
${ }^{7}$ An almost null open string trajectory attached to an AdS giant requires moving along the $\psi$ direction inside the $S^{5}$ and a direction parallel to the giant (see (3.6)). So, the present situation is somehow similar to the case of non-maximal giants in the $S^{5}$ [21, 25] and some exchange of angular momentum between the D-brane and the open string is expected.
} 
However, at the moment we do not know how to diagonalize the (2.29) and make an explicit comparison with (3.7). What we do know is the groundstate (2.30). Computing its mean occupation number is very elucidative,

$$
\frac{1}{L}\left\langle\Psi_{0}|\hat{n}| \Psi_{0}\right\rangle=\frac{1}{\alpha^{2}-1}=\frac{N}{p} .
$$

This computation gives, in average, the number of $Z$ fields in the word appended over the number of covariant derivatives. The former ones carry the R-charge identified in the dual description with angular momentum of the string along $\psi$. The covariant derivatives carry the spin charge identified in the dual description with angular momentum of the string along $\eta$.

Now, we can use the null trajectory (3.6) to compute the ratio of angular momentum components $J_{\psi}$ and $J_{\eta}$ of a massless particle traveling along it. The ratio of the angular momenta in both angular directions is

$$
\frac{J_{\psi}}{J_{\eta}}=\frac{1}{\sinh ^{2} \rho_{0}}=\frac{N}{p}
$$

where $p$ is the angular momentum of the giant defined in (3.5). This result coincides exactly with (3.8). Thus, the ground state (2.30) corresponds to the point like string. This enforces our interpretation of the first excitations of the Hamiltonian (2.29) as excitation modes of the open string in the pp-wave background.

\subsection{Semiclassical Open Strings}

Let us now consider a long open string ending on the AdS giant graviton, that feels the full $A d S_{5} \times S^{5}$ background. One can see that in the large angular momentum limit the system is well described by its classical action [31].

We concentrate on those coordinates subject to Dirichlet boundary conditions. We will eliminate the invariance of reparametrizations of the world-sheet by fixing a uniform gauge, instead of the conformal gauge [32]. This particular gauge turns out to be the appropriate one according to the particular labeling of the operators we used in the dual gauge theory.

We closely parallel the gauge fixing done in [21] and 25] for open strings ending on sphere giant gravitons. Let us begin by expressing the bosonic Polyakov action in terms of phase space variables. To do that, we use the conjugate momenta

$$
p_{\mu}=-G_{\mu \nu}\left(A \partial_{0} x^{\nu}+B \partial_{1} x^{\nu}\right)
$$

where $A=\sqrt{-g} g^{00}, B=\sqrt{-g} g^{01}$ and $g^{a b}$ is the worldsheet metric. The Polyakov action then takes the form

$$
S_{p}=\frac{1}{2 \pi \alpha^{\prime}} \int d \tau \int_{0}^{\pi} d \sigma \mathcal{L},
$$


where,

$$
\begin{aligned}
\mathcal{L} & =-\frac{1}{2} \sqrt{-g} g^{a b} G_{\mu \nu} \partial_{a} X^{\mu} \partial_{b} X^{\nu} \\
& =p_{\mu} \partial_{0} x^{\mu}+\frac{1}{2} A^{-1}\left[G^{\mu \nu} p_{\mu} p_{\nu}+G_{\mu \nu} \partial_{1} x^{\mu} \partial_{1} x^{\nu}\right]+B A^{-1} p_{\mu} \partial_{1} x^{\mu}
\end{aligned}
$$

$A$ and $B$ can be thought as Lagrange multipliers implementing the constraints

$$
\begin{aligned}
G^{\mu \nu} p_{\mu} p_{\nu}+G_{\mu \nu} \partial_{1} x^{\mu} \partial_{1} x^{\nu} & =0 \\
p_{\mu} \partial_{1} x^{\mu} & =0 .
\end{aligned}
$$

We will consider an open string moving with the giant graviton and along it. In terms of the global coordinates chosen in (3.1) the string will evolve only on $(t, \rho, \eta, \psi)$, i.e. the string is propagating on an $A d S_{3} \times S^{1} \subset A d S_{5} \times S^{5}$,

$$
d s^{2}=R^{2}\left(-\cosh ^{2} \rho d t^{2}+d \rho^{2}+\sinh ^{2} \rho d \eta^{2}+d \psi^{2}\right) .
$$

In these coordinates the position of the giant is given by

$$
\rho=\arg \cosh \sqrt{1+\frac{p}{N}}, \quad \psi=t .
$$

Now, we change to a coordinate system in which the giant gravitons is static

$$
r=\cosh \rho, \quad \phi=\psi-t
$$

We make this election of coordinates guided by the boundary conditions (2.43) and (2.44). The metric (3.15) becomes

$$
d s^{2}=R^{2}\left(-\left(r^{2}-1\right) d t^{2}+2 d t d \phi+d \phi^{2}+\frac{d r^{2}}{r^{2}-1}+\left(r^{2}-1\right) d \eta^{2}\right) .
$$

Calling $L$ the total angular momentum of the string along $\eta$

$$
L=\frac{1}{2 \pi \alpha^{\prime}} \int_{0}^{\pi} d \sigma p_{\eta}
$$

As originally done in [33], we choose a gauge in which $p_{\eta}$ is homogeneously distributed along the string, i.e. it is independent of $\sigma$. Moreover, we take $\tau$ to be coincident with the global time $t$,

$$
t=\tau, \quad p_{\eta}=2 \alpha^{\prime} L
$$

The election of distributing $p_{\eta}$ homogeneously is also inspired in the field theory analysis of the previous section. The spacing in the bosonic lattice was given by the covariant derivatives. So, the index $l$ running from 1 to $L$ in (2.29) counts uniformly covariant derivatives. Then, in the continuum limit, the action (2.42) has the spin 
charge associated to the covariant derivative uniformly distributed in the variable $\sigma$. This is why we expect the appearance of an action similar to (2.42) by fixing a gauge where $p_{\eta}$ is constant. On the contrary, if one worked with the labeling of the words as $\operatorname{sl}(2)$ spin chains, the discrete index of the chain would count uniformly scalar fields $Z$. In that case, the semiclassical action obtained projecting with $\operatorname{sl}(2)$ coherent states, would be similar to a Polyakov action in a gauge where the momentum $p_{\psi}$ is homogeneously distributed [28, 29].

The implementation of constraints (3.13) and (3.14) leads to a lagrangian of the form

$$
\mathcal{L}=V^{i} p_{i}-\sqrt{M^{i j} p_{i} p_{j}+M}
$$

where the indices $i, j=\phi, r$. The coefficients $V^{i}, M^{i j}$ and $M$ depend on the coordinates and their derivatives,

$$
\begin{aligned}
V^{\phi} & =\dot{\phi}+1 \\
V^{r} & =\dot{r} \\
M^{\phi \phi} & =r^{2}\left(1+\frac{R^{4}}{4 \alpha^{\prime 2} L^{2}}\left(r^{2}-1\right) \phi^{\prime 2}\right), \\
M^{\phi r} & =M^{r \phi}=r^{2}\left(r^{2}-1\right) \frac{R^{4}}{4 \alpha^{\prime 2} L^{2}} r^{\prime} \phi^{\prime}, \\
M^{r r} & =r^{2}\left(r^{2}-1\right)\left(1+\frac{R^{4}}{4 \alpha^{\prime 2} L^{2}} r^{\prime 2}\right), \\
M & =4 \alpha^{\prime 2} L^{2} \frac{r^{2}}{r^{2}-1}+R^{4} r^{2} \phi^{\prime 2}+R^{4} \frac{r^{2}}{r^{2}-1} r^{\prime 2} .
\end{aligned}
$$

As usual, dots and primes denote derivatives with respect to $\tau$ and $\sigma$ respectively. According to our conventions, $R^{4} / \alpha^{\prime 2}=\lambda / 8 \pi^{2}$. Also, recall that the coordinates $r$ and $\phi$ satisfy Dirichlet boundary conditions,

$$
\begin{aligned}
\left.r\right|_{\sigma=0, \pi} & =\sqrt{1+\frac{p}{N}}, \\
\left.\phi\right|_{\sigma=0, \pi} & =\text { const. }
\end{aligned}
$$

Variations of the momenta $p_{r}$ and $p_{\phi}$ give rise to algebraic equations of motion that can be used to solve the momenta,

$$
p_{i}=M_{i j} V^{j} \sqrt{\frac{M}{1-M_{k l} V^{k} V^{l}}},
$$

where $M_{i j}$ is the inverse of $M^{i j}$. With these expressions for the momenta, the Lagrangian can be written as

$$
\mathcal{L}=-\sqrt{\left(1-M_{i j} V^{i} V^{j}\right) M}
$$


Now, we assume that time derivatives are small. More precisely, we consider $\partial_{0} x^{\mu} \sim$ $\lambda / L^{2}$ and take this parameter $\lambda / L^{2} \ll 1$ [27]. For later convenience, we rescale $\sigma \rightarrow \sigma / \pi$ and obtain, to lowest order in the expansion on this small parameter, the following action

$$
S \approx-L \int d t \int_{0}^{1} d \sigma\left(\frac{\dot{\phi}}{r^{2}-1}-\frac{\lambda}{L^{2}}\left(r^{2}+r^{2} \phi^{2}\right)+\mathcal{O}\left(\frac{\lambda^{2}}{L^{4}}\right)\right) .
$$

Notice the factor $L$ in front of the action, which $L \rightarrow \infty$ in the limit we are considering. Then, it can play the rôle of the inverse of the Planck constant in a semiclassical limit.

Remarkably, (3.29) and boundary conditions (3.25)-(3.26) coincide with the semiclassical (2.42) action for the lattice Hamiltonian and boundary conditions (2.43)(2.44). Therefore, it is natural to identify coherent states parameters $u$ and $\phi$ with global coordinates $1 / \cosh \rho$ and $\psi-t$ respectively. Moreover, in this interpretation of the semiclassical coherent state action as the sigma model action corresponding to an open string, different labelings of the operators can be accounted by different ways of fixing the world-sheet reparametrization invariance of the Polyakov action.

\section{Discussion}

We have studied excited AdS giant gravitons and their gauge theory dual operators. We started describing the gauge invariant operators associated to open strings ending on AdS giant gravitons. To study their scale dimensions spectrum, we made use of the dilatation operator computed in [26] restricted to a $s l(2)$ sub-sector of the superconformal group. The mixing matrix of anomalous dimensions, at the one-loop approximation, corresponded to the Hamiltonian of an open $\operatorname{sl}(2)$ spin chain. The Hamiltonian included terms mixing spin chains of different lengths and at that point we introduced a labeling for the operators that enabled us to interpret the mixing matrix of anomalous dimensions as the Hamiltonian of a bosonic lattice. In analogy with the case of non-maximal sphere giants [21], the variability in the spin chain length was translated into a variable total number of bosons occupying the lattice. Interestingly, the Hamiltonian turned out to be non-quadratic in the lowering and raising operators and included non-nearest neighbor interactions. In spite of that, we showed that lattices with a large number of sites were effectively described by a local non-linear sigma-model action.

We were not able to compute the complete spectrum of the bosonic Hamiltonian. However, by a semiclassical analysis, similar to that of [25], we showed the existence of continuous bands. In fact, the variability of the total occupation number and

the existence of continuous bands prevented one to solve the problem in terms of a standard Bethe Ansatz. Interestingly, these continuous bands allow states with a 
monotonically growing number of bosons. The duals of such states correspond to open strings increasing monotonically their angular momentum at the expense of the giant. The analogy offered in [25] of an open string with accelerating end points also works here. The instability is due to the fact that for long enough strings, the tension will not support the weight due to the centrifugal force.

We have seen that the ratio between the $Z$ fields and covariant derivatives $\mathcal{D}$ mean values for the ground state of the Hamiltonian $(2.29)$ coincides exactly with the ratio between the angular momentum in the $\psi$ and $\eta$ directions for an unexcited open string spinning with and along the giant. This gives support to the proposed dual operators (2.10). In section 3 we have presented a semiclassical limit for the Polyakov action of open strings ending on AdS giant gravitons. We chose coordinates in which the giant is static, and fixed a gauge in which the string angular momentum along $\mathrm{AdS}, p_{\eta}$, is taken large and uniformly distributed along the string. In this gauge and in a rigid string limit, the Polyakov action and its Dirichlet boundary conditions ${ }^{8}$ coincided exactly with the non-linear sigma-model action and boundary conditions of the semiclassical description of the mixing matrix of anomalous dimensions. The agreement between these semiclassical actions is intuitively correct within the context of the AdS/CFT correspondence. Firstly, the string angular momentum $p_{\eta}$ taken large is the geometrical counterpart of the a large number of sites in the Hamiltonian. Secondly, the gauge choice that distributes $p_{\eta}$ uniformly is natural, since in the continuum limit the number of sites is uniformly rated by a continuous variable $\sigma$.

One of the motivations for studying AdS giant gravitons was that, in contrast to sphere giant gravitons, there is no upper bound for their angular momentum. In this regard, it is particularly interesting to consider the limit $\alpha \rightarrow \infty$ of the Hamiltonian (2.29). In that limit the Hamiltonian can be diagonalized perturbatively and the terms proportional to $\alpha^{2}$ in (2.29) serve to define a simple unperturbed Hamiltonian which, in our base, turns out to be diagonal. A one boson state in the $k^{\text {th }}$ site is an eigenstate with eigenvalue

$$
E_{k}^{0}=\lambda \alpha^{2} \frac{(L+1)}{k(L+1-k)}
$$

The first excitations of the unperturbed Hamiltonian are then of order $\lambda \alpha^{2} / L$. Although a perturbative treatment might be a fairly valid approximation for the large $\alpha$ limit of (2.29), it is clear that the one-loop approximation is no longer correct when taking $\lambda \rightarrow \infty$, as in the BMN limit. Thus, it would be inappropriate to compare the large $\alpha$ limit of the one-loop Hamiltonian, with any string theory result.

As a final comment, we would like to point out that the bosonic labeling developed in section 2 might be useful for studying other setups. Recently in [34], string bits in condensates of BPS configurations [35] and their relation to giant magnons

\footnotetext{
${ }^{8}$ We only consider string excitations along the directions subject to Dirichlet boundary conditions.
} 
[36], were conveniently characterized using a similar bosonic labeling for single trace operators in su(2) and su(3) sub-sectors. In terms of the coordinates and gauge choices appropriate for the bosonic labeling, the string bits or magnons are simply depicted as straight lines. We speculate that the bosonic labeling developed here might be useful to explore similar magnon excitations in the $\operatorname{sl}(2)$ sub-sector.

\section{Acknowledgements}

We would like to thank D.Berenstein for reading and commenting the manuscript. G.A.S. would like to thank the Physics group at CECS for financial support and warm hospitality. D.H.C. work was supported by Fondecyt grant 3060009 and G.A.S. work by CONICET grant PIP 6160. Institutional grants to CECS of the Millennium Science Initiative, Fundación Andes, and support by Empresas CMPC are also acknowledged.

\section{A. Conventions and some combinatorial properties}

We work with double line notation for the gauge theory. All fields are in the adjoint representation of the gauge group, e.g. $Z^{i}{ }_{j}=Z^{a}\left(T^{a}\right)^{i}{ }_{j}$, with $T^{a}$ the generators of $U(N)$ in the fundamental representation normalized as $\operatorname{tr}\left(T^{a} T^{b}\right)=\delta^{a b}$. The key identity is

$$
\left(T^{a}\right)_{j}^{i}\left(T^{a}\right)_{l}^{k}=\delta_{l}^{i} \delta_{j}^{k}
$$

We list below some properties of the totally symmetric tensor that are useful for the calculations of AdS giant gravitons. The totally symmetric tensor of rank $p$ is defined as

$$
S_{j_{1} \cdots j_{p}}^{i_{1} \cdots i_{p}} \equiv \sum_{\sigma} \delta_{j_{\sigma(1)}}^{i_{1}} \cdots \delta_{j_{\sigma(p)}}^{i_{p}}
$$

the summation being over all possible permutations $\sigma$. In (A.2), $p$ can be any integer and $i_{1}, \cdots, i_{p}$ and $j_{1}, \cdots, j_{p}$ range from 1 to $N$. Simple examples are

$$
\begin{aligned}
S_{j}^{i} & =\delta_{j}^{i} \\
S_{k l}^{i j} & =\delta_{k}^{i} \delta_{l}^{j}+\delta_{l}^{i} \delta_{k}^{j}
\end{aligned}
$$

Some useful properties of the $S$ tensors are

$$
\begin{aligned}
S_{j_{1} \cdots j_{p}}^{i_{1} \cdots i_{p}} & =\sum_{x=1}^{p} \delta_{j_{x}}^{i_{1}} S_{j_{1} \cdots j_{x-1} j_{x+1} \cdots j_{p}}^{i_{2}} \ldots i_{p} \\
S_{i_{1} \cdots i_{k} j_{k+1} \cdots j_{p}}^{i_{1} \cdots i_{k} i_{k+1} \cdots i_{p}} & =\frac{(N+p-1) !}{(N+p-1-k) !} S_{j_{k+1} \cdots j_{p}}^{i_{k+1} \cdots i_{p}} \\
S_{j_{1} \cdots j_{k}}^{i_{1} \cdots i_{k}} S_{l_{1} \cdots l_{p}}^{j_{1} \cdots j_{p}} & =k ! S_{l_{1} \ldots i_{k} j_{k+1} \cdots j_{p}}^{i_{1} \cdots l_{p}}
\end{aligned}
$$


Using the previous relations, it is possible to obtain the following contractions for two $S$ tensors,

$$
\begin{aligned}
S_{j_{1} \cdots j_{p-1} b}^{i_{1} \cdots i_{p-1} a} S_{i_{1} \cdots i_{p-1}}^{j_{1} \cdots j_{p-1} c} & =\frac{(N+p-1) !(p-1) !}{N !}\left(\delta_{b}^{a} \delta_{d}^{c}+\frac{(p-1)}{(N+1)} S_{b d}^{a c}\right) \\
S_{j_{1} \cdots j_{p-1} b h}^{i_{1} \cdots i_{p-1} a g} S_{i_{1} \cdots i_{p-1} d f}^{j_{1} \cdots j_{p-1} c e}= & \frac{(N+p-1) !(p-2) !}{(N+1) !}\left(S_{b h}^{a g} S_{d f}^{c e}+\frac{(p-2)(p-3)}{(N+2)(N+3)} S_{b h d f}^{a g c e}\right. \\
& \left.+\frac{(p-2)}{(N+2)}\left(\delta_{h}^{g} S_{b d f}^{a c e}+\delta_{b}^{g} S_{h d f}^{a c e}+\delta_{h}^{a} S_{b d f}^{g c e}+\delta_{b}^{a} S_{h d f}^{g c e}\right)\right)
\end{aligned}
$$

\section{B. Open strings in the pp-wave limit}

In this appendix we take a Penrose limit of the $A d S_{5} \times S^{5}$ geometry for the null geodesic: $\rho=\rho_{0}, t=\psi=\eta=\lambda$ discussed in eqn. (3.6). The limit is accomplished by defining a linear diffeomorphism to new coordinates and taking the $R \rightarrow \infty$ limit. The coordinate $u$ playing the rôle of parameter along the curve must appear, as discussed in section [3, with the same coefficient in $t, \psi$ and $\eta$. The remaining coefficients for the linear transformation can be fixed by demanding the metric to be well-defined in the $R \rightarrow \infty$ limit.

Consider the following change of coordinates,

$$
\begin{array}{lll}
t=u+\frac{v}{R^{2} \cosh ^{2} \rho_{0}}, & \rho=\rho_{0}+\frac{y}{R} \\
\psi=u-\frac{v}{R^{2} \cosh ^{2} \rho_{0}}-\tanh \rho_{0} \frac{x}{R}, & \theta=\frac{r}{R} \\
\eta=u-\frac{v}{R^{2} \cosh ^{2} \rho_{0}}+\frac{x}{R \cosh \rho_{0} \sinh \rho_{0}}, & \varphi=\frac{z}{R \sinh \rho_{0}} .
\end{array}
$$

keeping $\xi$ and $\Omega_{3}^{\prime}$ variables unchanged. After taking $R \rightarrow \infty$, the metric (3.1) becomes,

$$
d s^{2}=-4 d u d v+4 y d u d x-\left(r^{2}+z^{2}\right) d u^{2}+d x^{2}+d y^{2}+d z^{2}+z^{2} d \xi^{2}+d r^{2}+r^{2} d \Omega_{3}^{\prime 2} .
$$

In terms of cartesian coordinates

$$
\begin{array}{ll}
z_{1}=z \sin \xi, & z_{4}=r \sin \varphi^{\prime} \cos \xi^{\prime} \\
z_{2}=z \cos \xi, & z_{5}=r \cos \varphi^{\prime} \sin \eta^{\prime}, \\
z_{3}=r \sin \varphi^{\prime} \sin \xi^{\prime}, & z_{6}=r \cos \varphi^{\prime} \cos \eta^{\prime},
\end{array}
$$

the metric (B.2) takes the form

$$
d s^{2}=-4 d u d v+4 y d u d x-\sum_{a} z_{a}^{2} d u^{2}+d x^{2}+d y^{2}+\sum_{a} d z_{a}^{2} .
$$

This is the well known maximally supersymmetric pp-wave of type IIB supergravity

[4] displayed in unusual "magnetic" coordinates. This can be explicitly seen by an appropriate change of variables [37, 38]. 
The AdS giants attached strings that we consider have two different angular momenta: one related to the spinning along the $\psi$ direction of the $S^{5}$ called $J_{\psi}$ in (3.9) and a second one $J_{\eta}$ along the $\eta$ direction of the $S^{3} \subset A d S_{5}$ (see eqns.(3.1)-(3.2)). For weakly excited strings, the quantization on the pp-wave geometry (B.4) is a good approximation. We focus on the bosonic sector of the corresponding superstring action. In particular, on the excitation modes of coordinates $x$ and $y$ in (B.4) which satisfy Dirichlet boundary conditions.

The metric (B.4) coincides with the one considered in [25], so we can then borrow the complete analysis of oscillation modes and the canonical quantization done in it. The light-cone Hamiltonian expressed in terms of the oscillator operators $\beta_{m}, \tilde{\beta}_{m}, \beta_{m}^{\dagger}$ and $\tilde{\beta}_{m}^{\dagger}$ of the $x$ and $y$ coordinates is

$$
\begin{aligned}
H_{l c}^{x y} & =\frac{1}{8 \alpha^{\prime 2} p^{u}} \int_{0}^{2 \pi \alpha^{\prime} p^{u}} d \sigma\left(\dot{x}^{2}+\dot{y}^{2}+{x^{\prime}}^{2}+y^{\prime 2}\right) \\
& =\frac{1}{2 \alpha^{\prime} p^{u}} \sum_{n>0}\left(\omega_{n}^{-} \tilde{\beta}_{n}^{\dagger} \tilde{\beta}_{n}+\omega_{n}^{+} \beta_{n}^{\dagger} \beta_{n}\right),
\end{aligned}
$$

where $\omega_{n}^{ \pm}=\sqrt{\left(2 \alpha^{\prime} p^{u}\right)^{2}+n^{2}} \pm 2 \alpha^{\prime} p^{u}$. Thus, the energy of each excitation is

$$
\tilde{E}_{n}=\sqrt{1+\frac{n^{2}}{\left(2 \alpha^{\prime} p^{u}\right)^{2}}}-1, \quad E_{n}=\sqrt{1+\frac{n^{2}}{\left(2 \alpha^{\prime} p^{u}\right)^{2}}}+1 .
$$

To illuminate this result, we express the light-cone charges

$$
\begin{aligned}
H_{l c} & =-p_{u}=i \frac{\partial}{\partial u}, \\
p^{u} & =-\frac{1}{2} p_{v}=\frac{i}{2} \frac{\partial}{\partial v} .
\end{aligned}
$$

in terms of the conserved charges $\Delta, J_{\psi}$ and $J_{\eta}$ corresponding to the original global coordinates $t, \psi$ and $\eta$. The change of coordinates (B.1) gives,

$$
\begin{aligned}
H_{l c} & =i\left(\frac{\partial}{\partial t}+\frac{\partial}{\partial \psi}+\frac{\partial}{\partial \eta}\right)=\Delta-J_{\psi}-J_{\eta}, \\
p^{u} & =\frac{i}{2 R^{2} \cosh ^{2} \rho_{0}}\left(\frac{\partial}{\partial t}-\frac{\partial}{\partial \psi}-\frac{\partial}{\partial \eta}\right)=\frac{\Delta+J_{\psi}+J_{\eta}}{2 R^{2} \cosh ^{2} \rho_{0}} .
\end{aligned}
$$

To make contact with the gauge theory calculations, we denote

$$
\begin{aligned}
& J_{\eta}=L, \\
& J_{\psi}=\frac{L}{\sinh ^{2} \rho_{0}}=\frac{N}{p} L .
\end{aligned}
$$

$L$ denotes, on the gauge theory side, the total number of covariant derivatives present in the word $W$ (see eqn. (2.21)). The sum of angular momenta takes the form

$$
J_{\eta}+J_{\psi}=L\left(1+\frac{N}{p}\right)=L \frac{\alpha^{2}}{\alpha^{2}-1},
$$


where $\alpha$ is given by (2.17). To get a finite light-cone energy we required that $\Delta \simeq$ $\alpha^{2} L /\left(1-\alpha^{2}\right)$. Therefore,

$$
p^{u} \simeq \frac{L}{R^{2}\left(\alpha^{2}-1\right)}=\frac{L}{\sqrt{8 \lambda} \pi\left(\alpha^{2}-1\right)} .
$$

Here the relations $R^{4}=4 \pi g_{s} N \alpha^{\prime 2}$ and $\lambda=g_{s} N / 2 \pi$ have been used. Finally, the string excitation energies in terms of gauge theory parameters are

$$
\tilde{E}_{n}=\sqrt{1+\frac{2 \lambda \pi^{2}\left(\alpha^{2}-1\right)^{2} n^{2}}{L^{2}}}-1, \quad E_{n}=\sqrt{1+\frac{2 \lambda \pi^{2}\left(\alpha^{2}-1\right)^{2} n^{2}}{L^{2}}}+1 .
$$

When considering the BMN limit: $\lambda / L^{2}$ fixed and small as $L \rightarrow \infty$, the expansion of the square roots gives,

$$
\tilde{E}_{n} \approx \frac{\lambda \pi^{2}\left(\alpha^{2}-1\right)^{2} n^{2}}{L^{2}}, \quad E_{n} \approx 2+\frac{\lambda \pi^{2}\left(\alpha^{2}-1\right)^{2} n^{2}}{L^{2}}
$$

\section{References}

[1] J. M. Maldacena, "The large N limit of superconformal field theories and supergravity," Adv. Theor. Math. Phys. 2, 231 (1998) [Int. J. Theor. Phys. 38, 1113 (1999)] [arXiv:hep-th/9711200].

[2] S. S. Gubser, I. R. Klebanov and A. M. Polyakov, "Gauge theory correlators from non-critical string theory," Phys. Lett. B 428, 105 (1998) [arXiv:hep-th/9802109].

[3] E. Witten, "Anti-de Sitter space and holography," Adv. Theor. Math. Phys. 2, 253 (1998) [arXiv:hep-th/9802150].

[4] M. Blau, J. Figueroa-O'Farrill, C. Hull and G. Papadopoulos, "A new maximally supersymmetric background of IIB superstring theory," JHEP 0201, 047 (2002) [arXiv:hep-th/0110242].

[5] R. R. Metsaev, "Type IIB Green-Schwarz superstring in plane wave Ramond-Ramond background," Nucl. Phys. B 625, 70 (2002) [arXiv:hep-th/0112044].

[6] D. Berenstein, J. M. Maldacena and H. Nastase, "Strings in flat space and pp waves from N = 4 super Yang Mills," JHEP 0204, 013 (2002) [arXiv:hep-th/0202021].

[7] J. A. Minahan and K. Zarembo, "The Bethe-ansatz for $\mathrm{N}=4$ super Yang-Mills," JHEP 0303 (2003) 013. [arXiv:hep-th/0212208].

[8] N. Beisert and M. Staudacher, "The N = 4 SYM integrable super spin chain," Nucl. Phys. B 670, 439 (2003) [arXiv:hep-th/0307042].

[9] J. McGreevy, L. Susskind and N. Toumbas, "Invasion of the giant gravitons from anti-de Sitter space," JHEP 0006, 008 (2000) [arXiv:hep-th/0003075]. 
[10] M. T. Grisaru, R. C. Myers and O. Tafjord, "SUSY and Goliath," JHEP 0008, 040 (2000) [arXiv:hep-th/0008015].

[11] A. Hashimoto, S. Hirano and N. Itzhaki, "Large branes in AdS and their field theory dual," JHEP 0008, 051 (2000) [arXiv:hep-th/0008016].

[12] V. Balasubramanian, M. Berkooz, A. Naqvi and M. J. Strassler, "Giant gravitons in conformal field theory," JHEP 0204, 034 (2002) [arXiv:hep-th/0107119].

[13] S. Corley, A. Jevicki and S. Ramgoolam, "Exact correlators of giant gravitons from dual N = 4 SYM theory," Adv. Theor. Math. Phys. 5, 809 (2002) [arXiv:hepth/0111222].

[14] D. Berenstein, "Shape and holography: Studies of dual operators to giant gravitons," Nucl. Phys. B 675 (2003) 179 [arXiv:hep-th/0306090].

[15] D. Berenstein, "A toy model for the AdS/CFT correspondence," JHEP 0407, 018 (2004) [arXiv:hep-th/0403110].

[16] O. DeWolfe and N. Mann, "Integrable open spin chains in defect conformal field theory," JHEP 0404, 035 (2004) [arXiv:hep-th/0401041].

[17] K. Okamura, Y. Takayama and K. Yoshida, "Open spinning strings and AdS/dCFT duality," JHEP 0601, 112 (2006) [arXiv:hep-th/0511139].

[18] V. Balasubramanian, M. x. Huang, T. S. Levi and A. Naqvi, "Open strings from N = 4 super Yang-Mills," JHEP 0208, 037 (2002) [arXiv:hep-th/0204196].

[19] V. Balasubramanian, D. Berenstein, B. Feng and M. x. Huang, "D-branes in YangMills theory and emergent gauge symmetry," [arXiv:hep-th/0411205].

[20] D. Berenstein and S. E. Vazquez, "Integrable open spin chains from giant gravitons," JHEP 0506, 059 (2005) [arXiv:hep-th/0501078].

[21] D. Berenstein, D. H. Correa and S. E. Vazquez, "Quantizing open spin chains with variable length: An example from giant gravitons," Phys. Rev. Lett. 95, 191601 (2005) [arXiv:hep-th/0502172].

[22] R. de Mello Koch, N. Ives, J. Smolic and M. Smolic, "Unstable giants," Phys. Rev. D 73, 064007 (2006) [arXiv:hep-th/0509007].

[23] A. Agarwal, "Open spin chains in super Yang-Mills at higher loops: Some potential problems with integrability," arXiv:hep-th/0603067.

[24] K. Okamura and K. Yoshida, "Higher Loop Bethe Ansatz for Open Spin-Chains in AdS/CFT," arXiv:hep-th/0604100.

[25] D. Berenstein, D. H. Correa and S. E. Vazquez, "A study of open strings ending on giant gravitons, spin chains and integrability," arXiv:hep-th/0604123. 
[26] N. Beisert, "The complete one-loop dilatation operator of $\mathrm{N}=4$ super Yang-Mills theory," Nucl. Phys. B 676, 3 (2004) [arXiv:hep-th/0307015].

[27] M. Kruczenski, "Spin chains and string theory," Phys. Rev. Lett. 93, 161602 (2004) [arXiv:hep-th/0311203].

[28] B. J. Stefanski and A. A. Tseytlin, "Large spin limits of AdS/CFT and generalized Landau-Lifshitz equations," JHEP 0405, 042 (2004) [arXiv:hep-th/0404133].

[29] S. Bellucci, P. Y. Casteill, J. F. Morales and C. Sochichiu, "SL(2) spin chain and spinning strings on AdS(5) x S**5," Nucl. Phys. B 707, 303 (2005) [arXiv:hep-th/0409086].

[30] S. Bellucci, P. Y. Casteill and J. F. Morales, "Superstring sigma models from spin chains: The SU(1,1|1) case," Nucl. Phys. B 729, 163 (2005) [arXiv:hep-th/0503159].

[31] A. A. Tseytlin, "Semiclassical strings and AdS/CFT," arXiv:hep-th/0409296.

[32] M. Kruczenski, A. V. Ryzhov and A. A. Tseytlin, "Large spin limit of AdS(5) x S**5 string theory and low energy expansion of ferromagnetic spin chains," Nucl. Phys. B 692, 3 (2004) [arXiv:hep-th/0403120].

[33] G. Arutyunov and S. Frolov, "Integrable Hamiltonian for classical strings on AdS(5) x S**5," JHEP 0502 (2005) 059 [arXiv:hep-th/0411089].

[34] S. E. Vazquez, "BPS condensates, matrix models and emergent string theory," arXiv:hep-th/0607204.

[35] D. Berenstein, "Large N BPS states and emergent quantum gravity," JHEP 0601, 125 (2006) [arXiv:hep-th/0507203].

[36] D. M. Hofman and J. M. Maldacena, "Giant magnons," arXiv:hep-th/0604135.

[37] J. Michelson, "(Twisted) toroidal compactification of pp-waves," Phys. Rev. D 66, 066002 (2002) [arXiv:hep-th/0203140].

[38] M. Bertolini, J. de Boer, T. Harmark, E. Imeroni and N. A. Obers, "Gauge theory description of compactified pp-waves," JHEP 0301, 016 (2003) [arXiv:hep-th/0209201]. 